Claremont Colleges

Scholarship@ Claremont

All HMC Faculty Publications and Research

HMC Faculty Scholarship

10-1-1995

\title{
Strong X-ray Emission from High-Temperature Plasmas Produced by Intense Irradiation of Clusters
}

T. Ditmire

Lawrence Livermore National Laboratory

Thomas D. Donnelly

Harvey Mudd College

R. W. Falcone

University of California - Berkeley

M. D. Perry

Lawrence Livermore National Laboratory

\section{Recommended Citation}

T. Ditmire, T.D. Donnelly, R.W. Falcone, M.D. Perry, "Strong X-ray Emission from High-Temperature Plasmas Produced by Intense Irradiation of Clusters," Phys. Rev. Lett. 75, 3122 (1995). doi: 10.1103/PhysRevLett.75.3122

This Article is brought to you for free and open access by the HMC Faculty Scholarship at Scholarship @ Claremont. It has been accepted for inclusion in All HMC Faculty Publications and Research by an authorized administrator of Scholarship @ Claremont. For more information, please contact scholarship@cuc.claremont.edu. 


\title{
Strong X-Ray Emission from High-Temperature Plasmas Produced by Intense Irradiation of Clusters
}

\author{
T. Ditmire, ${ }^{1}$ T. Donnelly, ${ }^{2}$ R. W. Falcone, ${ }^{2}$ and M. D. Perry ${ }^{1}$ \\ ${ }^{1}$ Laser Program, Lawrence Livermore National Laboratory, P.O. Box 808, L-443, Livermore, California 94550 \\ ${ }^{2}$ Department of Physics, University of California at Berkeley, Berkeley, California 94720
}

(Received 14 June 1995)

\begin{abstract}
The interaction of an intense laser pulse with large $(\sim 100 \AA)$ clusters present in pulsed gas jets is shown to produce novel plasmas with electron temperatures far in excess of that predicted by abovethreshold ionization theory. The enhanced absorption of the laser light by the dense clusters results in the production of high ion charge states via collisional ionization resulting in strong $\mathrm{x}$-ray emission from the hot plasma.

PACS numbers: $52.50 . \mathrm{Jm}, 36.40 . \mathrm{Vz}, 52.25 . \mathrm{Nr}$
\end{abstract}

Much effort has gone into the understanding of the interaction of short $(\leq 1 \mathrm{ps})$, intense $\left(>10^{15} \mathrm{~W} / \mathrm{cm}^{2}\right)$ laser pulses with matter [1]. Many of these experiments have investigated the production of short wavelength radiation or the generation of energetic electrons. Substantial progress has been made in the generation of bright, coherent soft $\mathrm{x}$ rays in the 30 to $100 \mathrm{eV}$ range from underdense plasmas and neutral gases via harmonic generation [2] or x-ray lasers [3]. Incoherent emission extending to the megavolt range has also been observed from the interaction of these intense, short pulses with dense targets [4,5].

High pressure gas jets undergoing rapid expansion produce a unique combination of both gas and solid phase components providing an interesting medium for the study of high intensity laser-matter interactions [6]. Solid density clusters form in the jet, resulting from the cooling associated with the adiabatic expansion of the gas into vacuum. This cooling causes the gas to supersaturate and nucleate. Under appropriate conditions, when the gas jet stagnation pressure exceeds a few atmospheres, the clusters formed in the expanding jet can be quite large $\left(>10^{4}\right.$ atoms per cluster) for gases such as Ar, $\mathrm{Kr}, \mathrm{N}_{2}$, and $\mathrm{Xe}$ [6]. We have observed anomalous short wavelength emission from these gas jets when irradiated by an intense laser pulse with a duration less than the cluster disassembly time. We find that the production of the anomalous charge states is dominated by collisional heating of large clusters in the laser field during the laser pulse. Time resolved spectroscopy shows that this rapid heating is followed by $\mathrm{x}$-ray emission occurring on a relatively long time scale. In contrast with an earlier report [7], this emission is well described by emission in the underdense plasma that results after the heated clusters expand.

These experiments were motivated by the hypothesis that an adiabatically cooled gas jet might be expected to exhibit enhanced laser absorption relative to a conventional low density gas target due to the presence of solid density clusters. The effect would be significant only if the duration of the laser pulse was less than the expansion time of the cluster. During and after irradiation, the clus- ters will expand to produce a system with the radiative and kinetic properties of a bulk low density plasma. The presence of clusters has previously been shown to affect laser absorption on solid targets of porous Au black [8] and result in the emission of $\mathrm{x}$ rays from charge states in gas targets far beyond those which could be produced by above-threshold ionization (ATI) [9].

Up to an irradiance of approximately $10^{17} \mathrm{~W} / \mathrm{cm}^{2}$, absorption of femtosecond pulses in a low density gas is primarily through strong field ionization. Radiation emitted by the plasma following the laser pulse is well described by conventional radiative and three-body recombination into charge states produced by strong field ionization of individual atoms. The plasma temperature is determined by the single atom ATI energy distribution [10]. Only when the laser intensity approaches $10^{18} \mathrm{~W} / \mathrm{cm}^{2}$ do other heating mechanisms, such as stimulated Raman scattering, become important [11].

The presence of high density clusters makes inverse bremmstrahlung a significant method of absorption and heating due to the high collisionality within the cluster. If the cluster diameter is smaller than a skin depth, this heating occurs nearly uniformly throughout the cluster. Model calculations that include the effects of cluster expansion and electron free streaming during the laser pulse suggest that this mechanism dominates the absorption and heating of clusters with diameters in the 50$150 \AA$ A range when irradiated by femtosecond laser pulses. For a $130 \mathrm{fs}, 825 \mathrm{~nm}$ laser with peak intensity of between $10^{14}$ and $10^{17} \mathrm{~W} / \mathrm{cm}^{2}$, we find that the electron temperatures reach 1 to $2 \mathrm{keV}$ within the cluster. These hot electrons produce high charge states (e.g., $Z>8$ for Ar and $Z>10$ for $\mathrm{Kr}$ ) in the cluster by collisional ionization before it expands.

The hot cluster microplasmas will undergo rapid hydrodynamic expansion into the surrounding underdense plasma during and after the laser pulse. The time for the density within the cluster to drop from the solid density present initially $n_{0}$ to the surrounding ambient density $n_{s}$ is a measure of the expansion time of the cluster and can 
be estimated by assuming a sonic expansion. The resulting cluster expansion time is approximately

$$
\tau_{\mathrm{ex}} \approx r_{0} \sqrt{\frac{m_{i}}{Z k T_{e}}}\left(\frac{n_{0}}{n_{s}}\right)^{1 / 3},
$$

where $r_{0}$ is the initial cluster radius, $k T_{e}$ is the cluster electron temperature, and $m_{i}$ is the ion mass. For an argon cluster (initial lattice spacing $\sim 3.8 \AA$ ) with an initial radius of $50 \AA$, an initial electron temperature of $1 \mathrm{keV}$, a $Z \approx 8$, and a surrounding bulk plasma with a density of $10^{18}$ atoms $/ \mathrm{cm}^{3}$, the expansion time is approximately 1 ps. After the cluster expands, the plasma dynamics are determined by the underdense uniform plasma.

Our experiments were performed with a Cr:LiSAF laser that produces $0.5 \mathrm{~J}$ pulses at $825 \mathrm{~nm}$ with $130 \mathrm{fs}$ pulse duration [12]. The supersonic gas jet used in these experiments is a Mach 8 Laval nozzle that produces atom densities of $(1-5) \times 10^{18}$ atoms $/ \mathrm{cm}^{3}$ for backing pressures of 100 to $700 \mathrm{psi}$ [13]. For all experiments the laser was focused $\sim 1 \mathrm{~mm}$ from the nozzle output, which is $1.4 \mathrm{~cm}$ from the jet throat. We can estimate the extent of the atomic clustering for this jet from the empirical gas jet scaling parameter of Hagena $[14,15]$, which is given by

$$
\Gamma^{*}=k \frac{(d / \tan \alpha)^{0.85} p_{0}}{T_{0}^{2.29}},
$$

where $d$ is the jet throat diameter in $\mu \mathrm{m}(150 \mu \mathrm{m}$ for our jet), $\alpha$ is the jet expansion half angle $\left(\sim 5^{\circ}\right.$ for our jet), $p_{0}$ is the backing pressure in mbar, $T_{0}$ is the initial gas temperature ( $\sim 298 \mathrm{~K}$ for our experiments), and $k$ is an empirical constant $(k \approx 2900$ for $\mathrm{Kr}, 1700$ for $\mathrm{Ar}$, 180 for $\mathrm{Ne}$, and 4 for $\mathrm{He}$ [15]). Clustering begins when this parameter exceeds $\sim 300[6,14,16]$ and large clusters $\left(>10^{4}\right.$ atoms/cluster) predominate when $\Gamma^{*}>5 \times 10^{4}$ $[7,15,16]$. This parameter varied from $\sim 1 \times 10^{4}$ to $1 \times$ $10^{5}$ for $\mathrm{Ar}$ and $\sim 2 \times 10^{4}$ to $2 \times 10^{5}$ for $\mathrm{Kr}$ backing our jet with 100 to $600 \mathrm{psi}$, respectively. It never exceeded 5000 for $\mathrm{Ne}$ and $\mathrm{He}$ in our experiments.

We confirmed the presence of large clusters in this gas jet under standard operating conditions by a series of Mie scattering measurements. The gas at the output of the jet nozzle was irradiated with approximately $1 \mu \mathrm{J}$ of light from the frequency doubled LiSAF laser at $412 \mathrm{~nm}$. The centerline of the approximately $1 \mathrm{~mm}$ diameter flow was probed with a beam of approximately $400 \mu \mathrm{m}$ in diameter. The $90^{\circ}$ Mie scattered light was collected with a lens and imaged onto the face of a photomultiplier tube. Figure 1 shows the scattered light signal as a function of backing pressure for $\mathrm{He}, \mathrm{Ne}, \mathrm{Ar}$, and $\mathrm{Kr}$. No significant light scattering above the noise level is observed from either $\mathrm{Ne}$ or $\mathrm{He}$ over the range of backing pressures studied. The scattered light signal from the expansion of $\mathrm{Ar}$ and $\mathrm{Kr}$, however, exhibits nonlinear growth with backing pressure, rising above the noise with as little as 150 psi backing the gas jet. Assuming 100\% condensation of the atoms into clusters [17], the observed scattered signal levels at the highest backing pressures

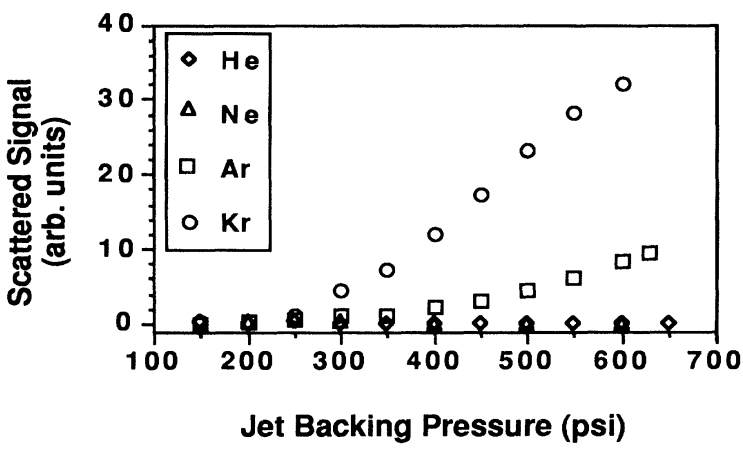

FIG. 1. Mie scattered light signal as a function of backing pressure for $\mathrm{He}$, $\mathrm{Ne}$, Ar, and $\mathrm{Kr}$.

suggest that the mean cluster size is $(2-10) \times 10^{4}$ atoms for $\mathrm{Ar}$ and $(1-5) \times 10^{5}$ atoms for $\mathrm{Kr}$, in good agreement with that expected from the clustering parameter, $\Gamma$.

Enhancement of the absorption of laser light by the presence of clusters was investigated by a series of experiments in which a small fraction $(0.01-0.1)$ of a gas known to cluster is mixed with $\mathrm{He}$ and passed through the gas jet. It is well known that the use of $\mathrm{He}$ as a carrier gas will significantly enhance the formation of clusters of heavier atoms in a gas expansion [17], while the He does not itself undergo clustering. The gas mixture was illuminated by the $130 \mathrm{fs}$ pulse focused by an $f / 25$ lens producing a peak intensity of up to $10^{17} \mathrm{~W} / \mathrm{cm}^{2}$. A grazing incidence extreme ultraviolet (XUV) spectrometer was used on the laser axis to disperse the resulting plasma emission. Time integrated XUV data were recorded with a microchannel plate detector and time resolved spectra were taken with an x-ray streak camera capable of $10 \mathrm{ps}$ time resolution.

Since the $\mathrm{He}$ does not itself cluster, observation of the intensity of the $\mathrm{Ly}-\alpha$ transition in $\mathrm{He}^{+}$at $304 \AA$ allowed us to study the dynamics of the bulk plasma without concern for intracluster effects. The presence of the clusters served to absorb laser energy, which resulted in a thermal plasma that can collisionally excite the $\mathrm{He}$ levels. Figure 2 shows the measured, time integrated intensity of the Ly- $\alpha$ line as a function of focused laser intensity for a variety of gas mixture conditions. The intensity required to optically ionize $\mathrm{He}$ to $\mathrm{He}^{2+}$ is approximately $1.5 \times 10^{16} \mathrm{~W} / \mathrm{cm}^{2}$ with a $130 \mathrm{fs}$ laser pulse. Above this intensity Ly- $\alpha$ light will be emitted due to the recombination of electrons into the upper levels of the doubly ionized He. As shown in Fig. 2, when a plasma is formed from $100 \%$ pure helium we observe a small amount of Ly- $\alpha$ light at peak intensities above the threshold for tunneling ionization to $\mathrm{He}^{2+}$. Addition of a small fraction of $\mathrm{Ne}$ does not significantly change the observed Ly- $\alpha$ signal. This is consistent with the fact that large clusters are absent in the $\mathrm{He}$ and $\mathrm{He} / \mathrm{Ne}$ expansions and the small observed signal is due only to direct strongfield ionization by the laser. 


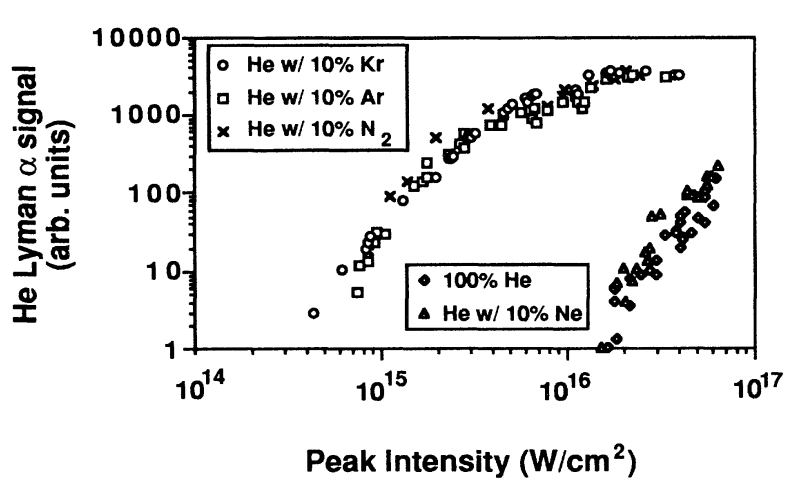

FIG. 2. Time integrated intensity of the He Ly- $\alpha$ line as a function of focused laser intensity for a variety of gas mixtures (350 psi gas jet backing pressure).

When a small amount of $\mathrm{Ar}, \mathrm{Kr}$, or $\mathrm{N}_{2}$ is mixed with the helium, all gases with a strong propensity for forming large clusters, the magnitude of the $\mathrm{He} \mathrm{Ly-} \alpha$ signal is significantly enhanced exceeding the signal of the pure helium by nearly a factor of 100 at the highest intensities. Furthermore, the Ly- $\alpha$ signal appears at an intensity that is 20 times lower than the threshold for the production of $\mathrm{He}^{2+}$ predicted by tunneling ionization. This large enhancement in Ly- $\alpha$ radiation can be explained by the creation of a hot plasma produced by efficient laser energy absorption into the dopant gas clusters. Time resolved data of the He Ly- $\alpha$ line (radiative lifetime $=133$ ps) indicate that the emission from the pure helium plasma decays after about $1.5 \mathrm{~ns}$, consistent with the relatively cold $(\sim 40 \mathrm{eV})$ optically ionized plasma predicted by tunnel ionization [10]. The emission from the plasma formed by irradiation of the gas mixtures lasts for approximately $150 \mathrm{~ns}$. This long-lived emission is evidence for a hot $(>100 \mathrm{eV})$ thermal plasma that is slowly cooling by hydrodynamic expansion.

The enhanced absorption and heating by clusters should lead to high charge states and short wavelength $(<100 \AA)$ $\mathrm{x}$ rays being produced at relatively modest laser intensity as described previously. A second grazing incidence spectrometer was used to measure x-ray emission above $100 \mathrm{eV}$ emitted $90^{\circ}$ to the laser propagation axis. Fig. 3(a) shows the time integrated spectrum between 40 and $100 \AA$ produced by the laser focused to an irradiance of approximately $1.5 \times 10^{16} \mathrm{~W} / \mathrm{cm}^{2}$ into the gas jet backed by $500 \mathrm{psi}$ of pure $\mathrm{Kr}$. The spectrum exhibits strong emission from the $4 p-3 d$ and $4 s-3 p$ arrays of $\mathrm{Kr}^{10+}, \mathrm{Kr}^{11+}, \mathrm{Kr}^{12+}$, and $\mathrm{Kr}^{13+}$ [18]. Tunnel ionization predicts that intensities of $3 \times 10^{17}, 4 \times 10^{17}, 6 \times 10^{17}$, and $8 \times 10^{17} \mathrm{~W} / \mathrm{cm}^{2}$, respectively, are required to produce these states by optical ionization, nearly an order of magnitude in excess of the actual laser irradiance present.

Anomalous lines from $\mathrm{Kr}^{10+}$ in this wavelength range were observed previously by McPherson et al. under similar conditions in a time integrated experiment using a $200 \mathrm{fs} \mathrm{KrF}, 248 \mathrm{~nm}$ laser to produce a plasma in
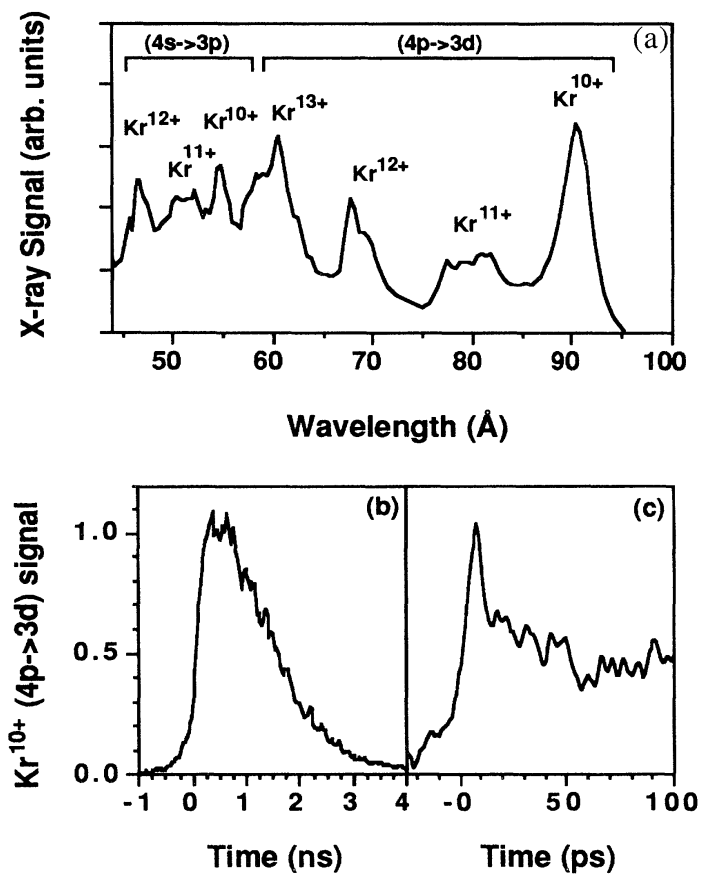

FIG. 3. (a) Time integrated spectrum produced in $\mathrm{Kr}$ (500 psi gas jet backing pressure) with an intensity of $1.5 \times 10^{16} \mathrm{~W} / \mathrm{cm}^{2}$. (b),(c) Measured time history of the $\mathrm{Kr}^{10+} 4 p-3 d$ line.

a $\mathrm{Kr}$ gas jet [7]. They attributed these lines to laser induced inner-shell excitation induced by excitation of small (few atom) clusters. This mechanism would produce radiation emitted on the time scale of the laser pulse $(<1000 \mathrm{fs})$. We find in our experiment that large clusters $\left(>10^{4}\right.$ atoms $)$ are present in the gas jet and that the emission from these lines is dominated by relatively long time scale processes (expansion, cooling, and recombination). The time history of the $\mathrm{Kr}^{10+} 4 p-3 d$ line is shown in Fig. 3(b) with a streak camera resolution of approximately 280 ps. The radiation from this line is emitted for nearly $3 \mathrm{~ns}$ after the passage of the laser through the gas jet. This lifetime is consistent with the long-lived emission of a hot, underdense bulk plasma.

Further evidence for the interaction of the laser with large solid density clusters is found when the time history of the $\mathrm{Kr}$ emission is observed over the first $100 \mathrm{ps}$. Figure 3(c) shows the time history of the $\mathrm{Kr}^{10+} 90 \AA$ line for the first $100 \mathrm{ps}$ after illumination by the laser. The time resolution here is $\sim 10 \mathrm{ps}$. We observe an initial spike in the emission of the $\mathrm{Kr}^{10+}$ line, faster than the time resolution of the streak camera, followed by the longlived emission demonstrated in Fig. 3(b). A similar spike is seen on all lines observed in Fig. 3(a). This initial spike is indicative of intense $\mathrm{x}$-ray emission by the dense cluster microplasma immediately after heating by the laser. This short time scale emission can be explained by enhanced collisional excitation within the hot plasma during the 
brief time in which the local density is high within the cluster. The fast $(<1 \mathrm{ps})$ expansion of the cluster is then followed by lower intensity emission by the lower density bulk plasma on a long (3 ns) time scale. We should emphasize that these data indicate that the vast majority of the x-ray emission comes not from the cluster but from the underdense plasma. The initial spike in the emission contains less than $1 \%$ of the total x-ray signal emitted from the plasma.

We have observed similar enhanced absorption and heating resulting in the production of high charge states and long-lived $\mathrm{x}$-ray emission in all species which undergo significant clustering. Figure 4(a) shows the time integrated spectrum of $\mathrm{Ar}$ heated by a pulse focused to $8 \times 10^{15} \mathrm{~W} / \mathrm{cm}^{2}$. Strong emission from lines below $50 \AA$ produce in neonlike $\operatorname{Ar}\left(\mathrm{Ar}^{8+}\right)$ are readily observed. To produce this charge state by tunnel ionization would require a focused intensity of $>1.5 \times 10^{18} \mathrm{~W} / \mathrm{cm}^{2}$, more than 2 orders of magnitude higher than that used in the experiment. These $250-400 \mathrm{eV}$ photons result from the formation of a hot plasma $\left(T_{e}>250 \mathrm{eV}\right)$. The measured photon yield on these lines indicates that up to $(2-5) \%$ of the laser energy is converted to $x$ rays in this wavelength range, yields that are comparable to solid targets [8]. Time resolved data on these lines exhibit similar behavior to that of the $\mathrm{Kr}$ lines, with the $\mathrm{Ar}^{8+} 49 \AA 3 s-2 p$ line, for example, lasting over 2 ns [Fig. 4(b)].

The mechanism of enhanced absorption in large, solid density clusters producing hot microplasmas and x-ray emission on a time scale determined by hydrodynamic expansion and cooling is supported by a one-dimensional hydrodynamic model and kinetics calculations of the low density plasma. We assume the cooling is determined by adiabatic expansion of a cylindrical plasma with corrections for conductive and free steaming cooling, and we use the kinetics code FLY [19] to determine the time history of various charge states. FLY solves rate equations to determine the time-dependent population of energy levels in the different ion species found in the plasma. We find that the observed charge states and resulting line time histories imply an initial plasma temperature

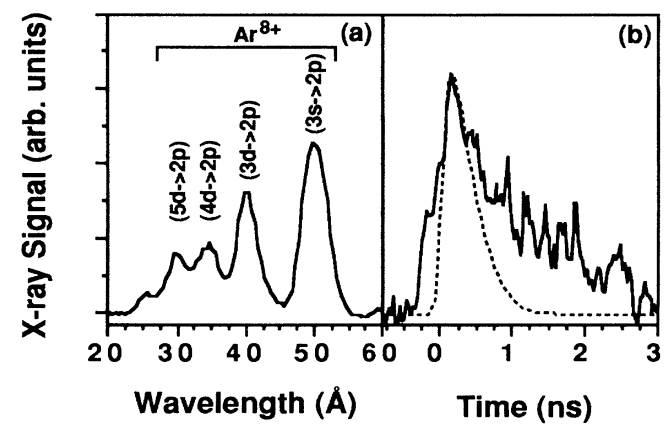

FIG. 4. (a) Time integrated spectrum produced in $\operatorname{Ar}(500$ psi gas jet backing pressure) with an intensity of $8 \times 10^{15} \mathrm{~W} / \mathrm{cm}^{2}$. (b) Measured (solid) and calculated (dashed) time history of the $\mathrm{Ar}^{8+} 49 \AA$ line. of $\sim 1000 \mathrm{eV}$ in the argon cluster. The calculated time history of emission on the $\mathrm{Ar}^{8+} 49 \AA$ line in a cylindrical plasma, with an initial electron temperature of $1000 \mathrm{eV}$ and a final ion density of $1 \times 10^{18} \mathrm{~cm}^{-3}$, is compared to the measured $49 \AA$ emission in Fig. 4. The calculated decay time is in reasonable agreement with the measured time history, predicting a long $(>1 \mathrm{~ns})$ decay consistent with the data.

In conclusion, we have shown that large clusters produced in expanding gas jets can be used to produce hot, moderate density plasmas with intense, short-pulse lasers. Enhanced absorption is observed when the pulse duration of the laser is less than the expansion time of the cluster. Plasmas containing charge states far beyond that predicted by optical field ionization of individual atoms are produced by the illumination of clusters by femtosecond pulses of $10^{16}$ to $10^{17} \mathrm{~W} / \mathrm{cm}^{2}$. The resulting strong $\mathrm{x}$-ray emission occurs on a time scale determined by hydrodynamic expansion and cooling of the plasma. Furthermore, the x-ray yields are comparable to those that can be achieved with solid targets. These cluster heated plasmas have the potential for providing a source of strong, $\mathrm{x}$-ray radiation with the modest irradiance $\left(10^{15}\right.$ to $10^{17} \mathrm{~W} / \mathrm{cm}^{2}$ ) produced by small-scale short-pulse lasers through a unique combination of the advantages inherent to both solid and gas targets.

We would like to acknowledge helpful discussions with R. A. Smith and the technical assistance of R. Jones. This work was supported by the AFOSR and the DOE under Contract No. W-7405-Eng-48.

[1] M. D. Perry and G. Mourou, Science 264, 917 (1994).

[2] A. L'Huillier et al., in Atoms in Intense Laser Fields, edited by M. Gavrila (Academic Press, Boston, 1992), p. 139.

[3] B.E. Lemoff et al., Phys. Rev. Lett. 74, 1574 (1995); Y. Nagata et al., Phys. Rev. Lett. 71, 3774 (1993).

[4] J. D. Kmetec et al., Phys. Rev. Lett. 68, 1527 (1992); A.P. Fews et al., Phys. Rev. Lett. 73, 1801 (1994).

[5] M. M. Murnane, H. C. Kapteyn, and R. W. Falcone, Phys. Rev. Lett. 62, 155 (1989).

[6] O.F. Hagena and W. Obert, J. Chem. Phys. 56, 1793 (1972).

[7] A. McPherson et al., Phys. Rev. Lett. 72, 1810 (1994).

[8] M. M. Murnane et al., Appl. Phys. Lett. 62, 1068 (1993).

[9] A. McPherson et al., Appl. Phys. B 57, 337 (1993).

[10] N. H. Burnett and P. B. Corkum, J. Opt. Soc. Am. B 6, 1195 (1989).

[11] W. J. Blyth et al., Phys. Rev. Lett. 74, 554 (1995).

[12] T. Ditmire and M. D. Perry, Opt. Lett. 18, 426 (1993).

[13] M. D. Perry et al., Opt. Lett. 17, 523 (1992).

[14] O. F. Hagena, Surf. Sci. 106, 101 (1981).

[15] J. Wörmer et al., Chem. Phys. Lett. 159, 321 (1989).

[16] V. M. Smirnov, Phys. Usp. 37, 621 (1994).

[17] B. J.C. Wu, P.P. Wegener, and G. D. Stein, J. Chem. Phys. 69, 1776 (1978).

[18] R. D. Bleach, J. Opt. Soc. Am. 70, 861 (1980).

[19] R. W. Lee, "User Manual for FLY" (unpublished). 